\title{
The role of self-reported olfactory and gustatory dysfunction as a screening criterion for suspected COVID-19
}

\author{
Liang En Wee ${ }^{1,2} \mathbb{D} \cdot$ Yvonne Fu Zi Chan ${ }^{2} \cdot$ Neville Wei Yang Teo $^{3} \cdot$ Benjamin Pei Zhi Cherng $^{2} \cdot$ Siew Yee Thien ${ }^{2}$. \\ Hei Man Wong ${ }^{2} \cdot$ Limin Wijaya ${ }^{2} \cdot$ Song Tar Toh ${ }^{3} \cdot$ Thuan Tong Tan ${ }^{2}$
}

Received: 14 April 2020 / Accepted: 18 April 2020 / Published online: 24 April 2020

○) Springer-Verlag GmbH Germany, part of Springer Nature 2020

Keywords Anosmia $\cdot$ Ageusia $\cdot$ COVID-19

Dear Editor,

We read with interest the paper written by Lechien et al. [1] describing a multi-centre cohort of COVID-19 patients, in which $85.6 \%$ had olfactory or taste disorders (OTD). If the association between COVID-19 and OTD is not recognized, the diagnosis may be missed. However, while a large proportion of COVID-19 patients had OTD, [1-3] the actual specificity as a criterion for screening suspected COVID-19 cases is unknown, as other viral infections may also cause OTD. Other studies have reported patients presenting with acute anosmia with or without upper respiratory tract symptoms, [4, 5] but not all patients were tested for COVID-19 [4]. Additionally, information on OTD in Asian cohorts is lacking [1]. In Singapore, the first case of COVID-19 was reported in end-January 2020. At our institution, the largest acute hospital in Singapore, from end-March 2020 all new admissions were screened for self-reported OTD at emergency department (ED) triage. All patients reporting new-onset OTD were admitted to exclude COVID-19. We

This comment refers to the article available online at https://doi. org/10.1007/s00405-020-05965-1.

Electronic supplementary material The online version of this article (https://doi.org/10.1007/s00405-020-05999-5) contains supplementary material, which is available to authorized users.

Liang En Wee

ian.wee@mohh.com.sg

1 Singhealth Infectious Diseases Residency, Singapore, Singapore

2 Department of Infectious Diseases, Singapore General Hospital, Singapore, Singapore

3 Department of Otolaryngology, Singapore General Hospital, Singapore, Singapore report our experience using self-reported OTD as a screening criterion for suspected COVID-19.

Over a 2-week period from 26 March-10 April 2020, given ongoing community transmission and the difficulty in distinguishing COVID-19 from ordinary viral infection, a questionnaire including respiratory symptoms, self-reported OTD, and travel and epidemiological risk factors was administered at ED triage to risk-stratify admissions. Suspect case criteria for COVID-19 were defined as the presence of respiratory symptoms and suspicious epidemiological links or travel history; or new-onset OTD. Testing was not limited to hospitalised inpatients; as part of the national strategy of containment, all patients who fulfilled suspect case criteria for COVID-19 were tested via real-time reverse transcription PCR (rRT-PCR) of oropharyngeal swabs, even if hospitalization was not otherwise required. Inpatient, if patients had respiratory symptoms and a viral prodrome, or OTD, oropharyngeal specimens were also tested for a routine panel of respiratory viruses. As this study was based on aggregated surveillance data, ethics approval was not required under our hospital's Institutional Review Board guidelines.

Over the study period, a total of 870 patients fulfilled suspect case criteria for COVID-19 at ED triage. A minority $(5.05 \%, 44 / 870)$ presented with OTD. The majority of suspects $(65.3 \%, 568 / 870)$ were well and discharged to selfisolate while awaiting results. Amongst suspected COVID19 cases, 154 patients $(17.9 \%, 154 / 870)$ tested positive. Of those, roughly one-fifth $(22.7 \%, 35 / 154)$ had OTD. The presence of OTD had high specificity as a screening criterion for COVID-19 (98.7\%, 95\% CI 97.6-99.4\%), but lower sensitivity $(22.7 \%, 95 \%$ CI $16.4-30.2 \%)$. This was roughly equivalent to the specificity and sensitivity of a history of close contact with a confirmed COVID-19 case (specificity: $94.8 \%, 95 \%$ CI $93.0-96.3 \%$; sensitivity: $27.3 \%, 95 \%$ CI 20.4-35.0\%), Supplementary Table 1. Amongst the 35 
COVID-19 positives with concomitant OTDs, three presented with isolated anosmia without other symptoms. Fever was the most common concomitant symptom $(21 / 35$, $60.0 \%)$, followed by cough $(10 / 35,28.5 \%)$ and rhinorrhea $(10 / 35,28.5 \%)$. Over the same period, a total of 71 admitted inpatients tested positive for other respiratory viruses, of which the most common was rhinovirus $(53.5 \%, 38 / 71)$; followed by influenza $(21.1 \%, 15 / 71)$, adenoviruses $(8.5 \%$, $6 / 71)$, other coronaviruses $(7.0 \%, 5 / 71)$. Only $2.8 \%(2 / 71)$ self-reported OTD (one rhinovirus, one human coronavirus 229E). Amongst patients with PCR-proven acute respiratory viral infections, those positive for COVID-19 had higher odds of OTD compared to those positive for other respiratory viruses (odds ratio, $\mathrm{OR}=10.14,95 \%$ CI 2.37-43.49, $p<0.001)$.

In conclusion, self-reported OTD had high specificity as a screening criterion for COVID-19 in an Asian cohort. Patients with COVID-19 appeared to have higher odds of OTD compared to those positive for other respiratory viruses. Routine screening in patients with new-onset OTD can improve case detection during a COVID-19 outbreak.

\section{Compliance with ethical standards}

Conflict of interest The authors report no conflicts of interest.

\section{References}

1. Lechien JR, Chiesa-Estomba CM, De Siati DR et al (2020) Olfactory and gustatory dysfunctions as a clinical presentation of mildto-moderate forms of the coronavirus disease (COVID-19): a multicenter European study. Eur Arch Otorhinolaryngol. https://doi. org/10.1007/s00405-020-05965-1 (Epub ahead of print)

2. Giacomelli A, Pezzati L, Conti F et al (2020) Self-reported olfactory and taste disorders in SARS-CoV-2 patients: a cross-sectional study. Clin Infect Dis. https://doi.org/10.1093/cid/ciaa330

3. Vaira LA, Salzano G, Deiana G, De Riu G (2020) Anosmia and ageusia: common findings in COVID-19 patients. Laryngoscope. https://doi.org/10.1002/lary.28692 (Epub ahead of print)

4. Hopkins C, Surda P, Kumar N (2020) Presentation of new onset anosmia during the COVID-19 pandemic. Rhinology. https://doi. org/10.4193/Rhin20.116 (Epub ahead of print)

5. Gane SB, Kelly C, Hopkins C (2020) Isolated sudden onset anosmia in COVID-19 infection: a novel syndrome? Rhinology. https ://doi.org/10.4193/Rhin20.114 (Epub ahead of print)

Publisher's Note Springer Nature remains neutral with regard to jurisdictional claims in published maps and institutional affiliations.

Funding This work was not grant-funded. 